\title{
Pulsar timing array
}

\author{
Alexander E. Rodin \\ Pushchino Radio Astronomy Observatory of the Lebedev Physical Institute, \\ Pushchino, Moscow region, 142290, Russia \\ email: rodin@prao.ru
}

\begin{abstract}
Simultaneous timing of several pulsars distributed over the sky, so called Pulsar Timing Array (PTA), is used for a variety of metrological and astronomical applications. Three examples of PTA application are presented: link between celestial reference frames, ensemble pulsar time scale and detection of gravitational waves.
\end{abstract}

Keywords. pulsar timing, reference systems, time, gravitational waves

Pulsar timing coordinates, which relates with the reference frame based on the planetary ephemeris (e.g. DE200, DE405), when combining with the pulsar VLBI positions based on the coordinates of the extragalactic radio sources (ICRF) gives possibility to link the reference frames. The rotation angles between DE200 and ICRF were calculated on the basis of pulsar VLBI observations (Rodin \& Sekido 2002): $A_{x}=-4 \pm 2$, $A_{y}=-13 \pm 3, A_{z}=-17 \pm 5$ mas.

The algorithm of the ensemble pulsar time $\mathrm{PT}_{\text {ens }}$ based on the optimal Wiener filtration method has been proposed in the paper (Rodin 2008). The method has been applied to the timing data of millisecond pulsars PSR J0613-0200, J1640+2224, J1643-1224, $\mathrm{J} 1713+0747, \mathrm{~B} 1855+09, \mathrm{~B} 1937+21$ and J2145-750. Fractional instability of TT-PT $\mathrm{P}_{\text {en }}$ is equal to $\sigma_{z}=(0.8 \pm 1.9) \cdot 10^{-15}$. A new limit of the energy density of the gravitational wave background based on $\sigma_{z}\left(\mathrm{TT}-\mathrm{PT}_{\mathrm{ens}}\right)$ was calculated to be equal to $\Omega_{g} h^{2} \sim 3$. $10^{-9}$.

Detection of gravitational waves with PTA is based on analysis of the angular correlation function (two-point correlation) of the post-fit pulsar timing residuals (Jenet et al. 2005). Direct use of pulsar time of arrivals (TOAs) is complicated by the fact that they contain contribution of different kinds of noise, which has different amplitudes and begins to dominate at various time intervals. Pulsar timing data were expanded into components

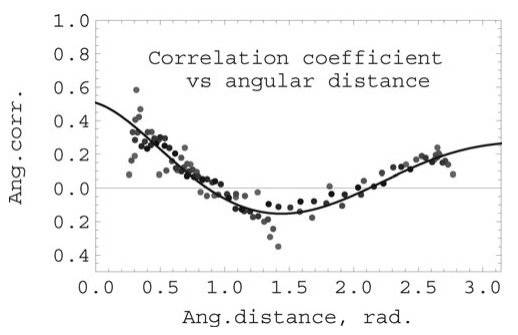

by model independent Caterpillar-SSA method (Golyandina et al. 2001). Two-point correlation was calculated for each component. Some components showed distinct angular correlation function (see fig.) which agrees well with the theoretically predicted one: correlation coefficient between theoretical and experimental angular correlation functions is equal to $\rho=0.94 \pm 0.08$. 


\section{References}

Golyandina, N., Nekrutkin, V., \& Zhigljavsky, A., Analysis of Time Series Structure SSA and Related Techniques. CHAPMAN \& HALL/CRC, 2001

Jenet, F., Hobbs, G., Lee, K. J., \& Manchester, R. N., 2005, ApJ, 625L, 123

Rodin, A. E. \& Sekido, M. 2002, in E. Ros, R. W. Porcas, A. P. Lobanov, \& J. A. Zensus (eds.), Proc. 6th European VLBI Network Symposium (Bonn, Germany), p. 247

Rodin, A. E., 2008, MNRAS, 387, 1583-1588 